\title{
Towards a structurally resolved human protein interaction network
}

David F. Burke ${ }^{1^{*}}$, Patrick Bryant ${ }^{2,3^{*}}$, Inigo Barrio-Hernandez ${ }^{1^{*}}$, Danish Memon ${ }^{1^{*}}$, Gabriele Pozzati $^{2,3^{*}}$, Aditi Shenoy ${ }^{2,3}$, Wensi Zhu ${ }^{2,3}$, Alistair S Dunham ${ }^{1}$, Pascal Albanese ${ }^{4.5}$, Andrew Keller $^{6}$, Richard A. Scheltema ${ }^{4,5}$, James E. Bruce ${ }^{6}$, Alexander Leitner ${ }^{7}$, Petras Kundrotas ${ }^{1,8 \#, ~}$ Pedro Beltrao" ${ }^{1 \#}$, Arne Elofsson",3\#.

1 - European Molecular Biology Laboratory, European Bioinformatics Institute (EMBL-EBI), Cambridge, UK

2 - Science for Life Laboratory, Stockholm University 17221 Solna, Sweden

3 - Department of Biochemistry and Biophysics, Stockholm University, 10691 Stockholm, Sweden

4 - Biomolecular Mass Spectrometry and Proteomics, Bijvoet Center for Biomolecular Research and Utrecht Institute of Pharmaceutical Sciences, Utrecht University, 3584 Utrecht, The Netherlands

5 - Netherlands Proteomics Center, 3584 Utrecht, The Netherlands

6 - Department of Genome Sciences University of Washington Seattle WA 98109

7 - Department of Biology, Institute of Molecular Systems Biology, ETH Zurich, Otto-Stern-Weg 3, 8093 Zurich, Switzerland

8 - Center for Computational Biology, The University of Kansas, Lawrence, KS 66047, USA

* Contributed equally

\# Correspondence to: pkundro@ku.edu, pbeltrao@ebi.ac.uk, arne@bioinfo.se

\section{Abstract}

All cellular functions are governed by complex molecular machines that assemble through protein-protein interactions. Their atomic details are critical to the study of their molecular mechanisms but fewer than $5 \%$ of hundreds of thousands of human interactions have been structurally characterized. Here, we test the potential and limitations of recent progress in deep-learning methods using AlphaFold2 to predict structures for 65,484 human interactions. We show that higher confidence models are enriched in interactions supported by affinity or structure based methods and can be orthogonally confirmed by spatial constraints defined by cross-link data. We identify 3,137 high confidence models, of which 1,371 have no homology to a known structure, from which we identify interface residues harbouring disease mutations, suggesting potential mechanisms for pathogenic variants. We find groups of interface phosphorylation sites that show patterns of co-regulation across conditions, suggestive of coordinated tuning of multiple interactions as signalling responses. Finally, we provide examples of how the predicted binary complexes can be used to build larger assemblies. Accurate prediction of protein complexes promises to greatly expand our understanding of the atomic details of human cell biology in health and disease. 


\section{Introduction}

Proteins are key cellular effectors determining most cellular processes. These rarely act in isolation, but instead, the coordination of the diversity of processes arises from the interaction among multiple proteins and other biomolecules. The characterization of protein-protein interactions is crucial for understanding which groups of proteins form functional units and underlies the study of the biology of the cell. Diverse experimental and computational approaches have been developed to determine the protein-protein interaction network of the cell (i.e. the interactome) with hundreds of thousands of human protein interactions determined to date (Orchard et al, 2014; Luck et al, 2020; Drew et al, 2021). These interactions vary from transient interactions that can regulate an enzyme to permanent interactions in large molecular machines.

The structural characterisation of any interactome has lagged behind its experimental determination due to technical limitations, with experimental and homology models currently covering an estimated 15 thousand human interactions (Mosca et al, 2012; Burley et al, 2021). The structural characterisation of protein complexes is a critical step in understanding the mechanisms of protein function, studying the impact of natural and disease mutations (Mosca et al, 2012; Wang et al, 2012; Kamburov et al, 2015; Porta-Pardo et al, 2015) and the regulation of cellular processes via the post-translational tuning of binding affinities (Beltrao et al, 2012; Nishi et al, 2011; Šoštarić et al, 2018; Betts et al, 2017).

While there has been great progress in experimental techniques for determining large complexes, current experimental approaches are not easily scalable. Computational approaches for predicting the structure of interactions on a large scale have relied primarily on identifying structural similarity for pairs of proteins against experimentally determined protein complexes (Zhang et al, 2012; Mosca et al, 2012; Wang et al, 2012; Mosca et al, 2014). Based on these approaches, the Interactome 3D repository currently lists 7625 predicted models based on homology, a number similar to the 8359 interactions listed in this resource as having an experimentally determined model. In addition to modelling based on homology, co-evolution based information has been used to predict interaction pairs and guide structural docking for bacterial proteins (Cong et al, 2019). More recently, neural network-based approaches have demonstrated the ability not only to accurately predict structures of individual proteins (Baek et al, 2021; Jumper et al, 2021) but also the structure of protein complexes (Bryant et al, 2021; Pozzati et al; Baek et al, 2021; Akdel et al, 2021; Evans et al, 2021). In benchmark sets where protein pairs are known to form a direct complex, these approaches can correctly predict the structure of up to $60 \%$ of the dimers (Bryant et al, 2021). These methods have been recently used to predict structures of 1,506 S. cerevisiae interactions that were selected based on co-evolution signals (Humphreys et al, 2021). However, the application of these neural network models for the large-scale prediction of human complex structures has not been tested yet.

Here, we assess the possibilities and limitations of applying AlphaFold2 to modelling human interactions on a large scale. We predicted the complex structures for two sets of human interactions obtained using different experimental methods, comprising 65,484 unique human 
interactions. Metrics derived from the predicted structures can be used to rank the models according to confidence, with 3,137 predicted structures ranked as highly confident. Interactions supported by a combination of methods indicating a high affinity and direct interaction result in higher confidence predictions, and higher confidence models are more likely to be supported by constraints indicated by orthogonal cross-link data. We showcase the value of a structurally resolved interactome by studying disease mutations and phosphorylation of interface residues. Finally, we provide some indication that binary complexes can be used to build higher-order assemblies.

\section{Results}

\section{Structure prediction of high confidence human protein interactions}

We selected experimentally determined human interactions from the Human Reference Interactome (HuRI) and the Human Protein Complex Map (hu.MAP 2.0). HuRI comprises interactions determined by yeast two-hybrid screening (Luck et al, 2020) from which we modelled 55586 pairs. From hu.MAP we selected 10,207 high-quality (confidence score $\geq 0.5$ ) protein-protein interactions (PPIs), which were derived by integration of affinity purification, co-fractionation and proximity ligation assays (Drew et al, 2021). While HuRI is more likely to be enriched for direct protein interactions, including potentially transient partners, the hu.MAP set is more likely to reflect stable protein interactions, including members of the same complex that may not necessarily interact directly. The overlap between the two datasets is small (309 pairs), and a comparison with two large scale compendiums of structural models ((Mosca et al, 2012), Methods) indicates that 62,019 of the combined pairs do not have experimental models or can be modelled easily by homology, suggesting a significant potential gain in structural knowledge.

We predicted the structure of 65,484 non-redundant pairs using the FoldDock pipeline (Bryant et al, 2021), based on AlphaFold2 (Jumper et al, 2021). We have previously shown that larger interface size and higher IDDT (pIDDT) scores from AlphaFold2 in the interfaces of the predicted complexes are associated with more reliable predictions (Bryant et al, 2021). As in the FoldDock pipeline, we combined these two metrics into a single score, which can be used to predict the DockQ score of a complex, dubbed pDockQ (Methods) that can rank models by confidence. We tested the overlap performance and ranking by pDockQ score by comparing the predicted models with experimental models. Across 1,465 comparisons, 742 (50\%) of predicted complexes were deemed to be well modelled (DockQ>0.23). For predictions with $p D o c k Q>0.23$, we estimate that $70 \%$ (671 out of 955) are well modelled and for pDockQ>0.5, 80\% (521 out of 651) are considered well modelled. However, it is worth noting that this estimated performance applies to cases where we expect that two proteins interact via direct contact in a single conformation.

We show in Fig. 1A the distribution of $p D o c k Q$ for the predicted interactions and a set of predicted structures for 2,000 random pairs of proteins. The pDockQ of known interacting proteins tends to be higher than for the random set with the predictions for hu.MAP showing on 
average higher confidence than for the HuRI set. Additionally, when selecting hu.MAP interactions also supported by yeast-two-hybrid $(\mathrm{Y} 2 \mathrm{H})$ or cross-link data (cross linking) results in much higher confidence values (Fig. 1A). This suggests that high confidence models are enriched for protein interactions supported by the two types of methods associated with high affinity and direct interactions. Based on the benchmarking results, we selected 3,137 structures (Fig. 1B) as high confidence models based on a cutoff of $p D o c k Q>0.5$, which would indicate around $80 \%$ of correct models in comparison with the experimental models. Only $0.3 \%$ of the random set of models would be considered a confident prediction at this cut-off. In Fig. 1C we show examples of predicted structures aligned to experimental or homology models, showing how the predictions and the confidence score relate to the observed alignments. For the majority of these cases, even with lower confidence values, the interaction interface is generally in good agreement, except for the PSMC2-PSMD11 interaction, which has the lowest confidence score of the illustrated models.

A list of protein interactions with predicted structural models, confidence metrics and annotations are provided in Table $\mathbf{S 1}$ and all models are available as described in the data availability section.

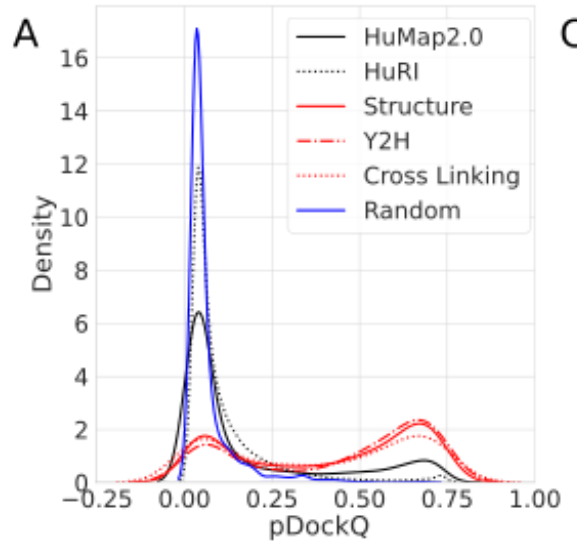

C

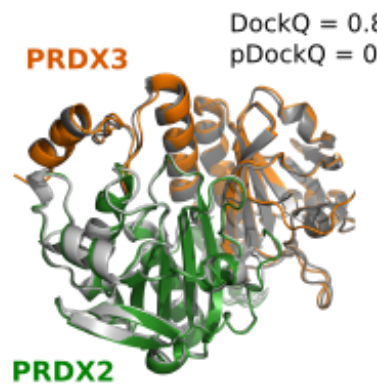

PRDX2
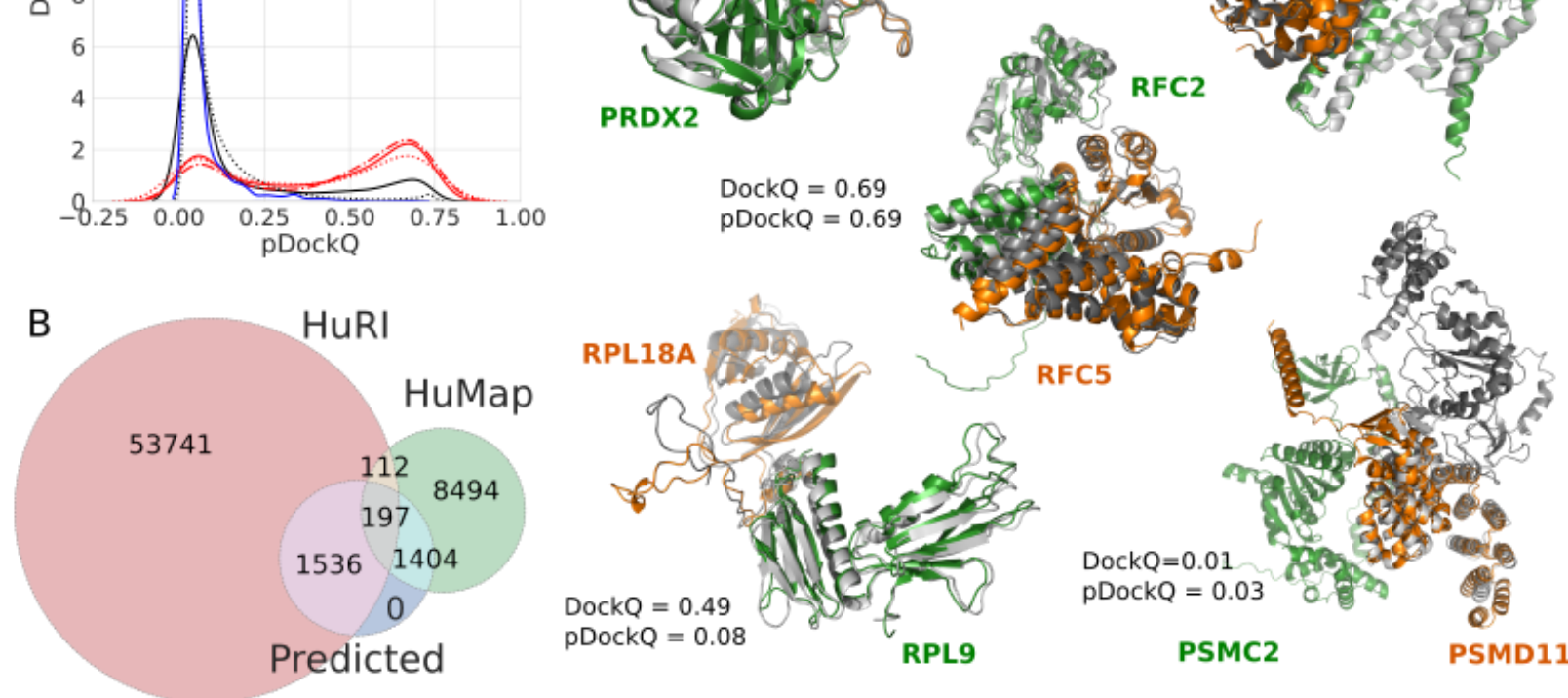

DockQ $=0.01$

$\mathrm{pDockQ}=0.03$

PSMC2

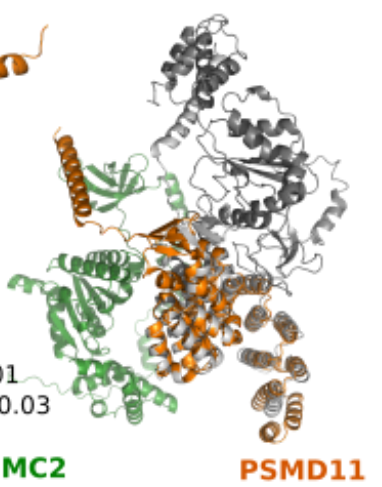

Figure 1 - Application of Alphafold2 complex predictions to a large dataset of human protein-protein interactions. A) Distribution of model confidence score ( $p D o c k Q)$ for predicted structures from two large human protein interaction datasets (hu.MAP and HuRI), compared with confidence metrics from 2000 random pairs of proteins. The hu.MAP dataset was further subsetted to those that have support from yeast two-hybrid (Y2H), cross-link data 
(Cross-linking) or correspond to pairs with available experimental or homology modelling information (Structure). B) Number interactions with models built from both datasets and those that we consider being of high confidence (Predicted), corresponding to those with pDockQ>0.5. C) Examples of predicted models (orange and green) overlapped with the corresponding experimental models (grey) and the observed (DockQ) or predicted ( $p D o c k Q)$ quality of the models.

\section{Cross-linking support for predicted complex structures}

Chemical cross-linking followed by mass spectrometry is an approach that can be used to identify reactive residues (usually lysines) that are in proximity, limited by the size of the cross-link agent used. The identification of such residues across a pair of proteins can help define the likely protein interface. To determine if the predicted complex structures agree with such orthogonal spatial constraints, we obtained a compilation of cross-links for pairs of residues across 528 protein pairs with predicted models (Fig. 2A, Table S1, see Methods). Of these, $51 \%$ of the pairs have one or more cross-links at a distance below the expected maximal distance possible (Fig. 2A). Restricting the predicted models to higher confidence by the pDockQ score increases the fraction complexes with cross-links within the maximal distance possible, reaching $75 \%$ for $\mathrm{pDockQ}$ scores greater than 0.5 (Fig. 2A). This result is in line with the benchmark results above, indicating that most models are likely to be correct at a pDockQ cut-off above 0.5 . Additionally, predicted structures with $p D o c k Q>0.23$ are also likely to have many correct models as judged by the fraction supported by cross-linking.

In total, we have identified 479 cross-links providing supporting evidence for 171 predicted complex structures with $\mathrm{pDockQ}>0.5$. Out of these, 41 correspond to complex structures with no experimental structure or homology models, from which we selected some to illustrate in Fig. 2B-E. Fig. 2B shows the AF2 model for the full length of the ERLIN1/ERLIN2 complex, which mediates the endoplasmic reticulum-associated degradation (ERAD) of inositol 1,4,5-trisphosphate receptors (IP3Rs). Alphafold2 predicts a globular domain (1-190) followed by an extended helical region with a kink around amino acid position 280 . Unlike the model in the interactome3D, the paralogous proteins are stacked side-by-side with the hydrophobic rich face of the helices buried and the hydrophilic face (mainly Lys) exposed to solvent. A cross-link between the C-terminal residues K275 (ERLIN1) and K287 at $18 \AA$, supports the predicted model. In Fig. 2C we show the model for proteins IMMT and $\mathrm{CHCHD3,} \mathrm{components} \mathrm{of} \mathrm{the}$ mitochondrial inner membrane MICOS complex. Alphafold2 predicts a globular helical domain at the C-terminal end of IMMT (550-750) to interact with the C-terminal end of CHCHD3 (150-225). This is supported by data of three cross-links between; K173 (CHCD3) and K565 (IMMT), and K203 (CHCD3) to both K714 and K726 of IMMT. Fig. 2D shows the complex of tRNA-guanine-N(7)-)-methyltransferase (METTL) with its non-catalytic subunit (WDR4). The structure of WDR4 has not yet been solved experimentally but contains WD40 repeats, which are expected to form a $\beta$-propeller domain, as predicted here. The METTL domain is predicted to interact with the side of the WDR40, away from the ligand-binding pore. This orientation is supported by a cross-link between K122 (WDR4) and K143 (METTL) (18Å). Finally, in Fig. 2E 
we show the predicted complex structure for the Heterogeneous nuclear ribonucleoproteins $\mathrm{C}$ (HNRNPC) and the RNA-binding protein, Raly. Two regions in both proteins are predicted with high confidence ( $p L D D T>70$ ), with the lower confidence regions not shown. The N-terminal domain in HNRNPC (16-85) is predicted to interact with the N-terminal domain of RALY (1-100). A long helix in HNRNPC (185-233) is predicted to interact with a helix in RALY (169-228). This interhelix interface is supported by crosslinking data for three pairs of lysines at either end of the helices $(189 \rightarrow 222 ; 229 \rightarrow 179$ and $232 \rightarrow 183)$.
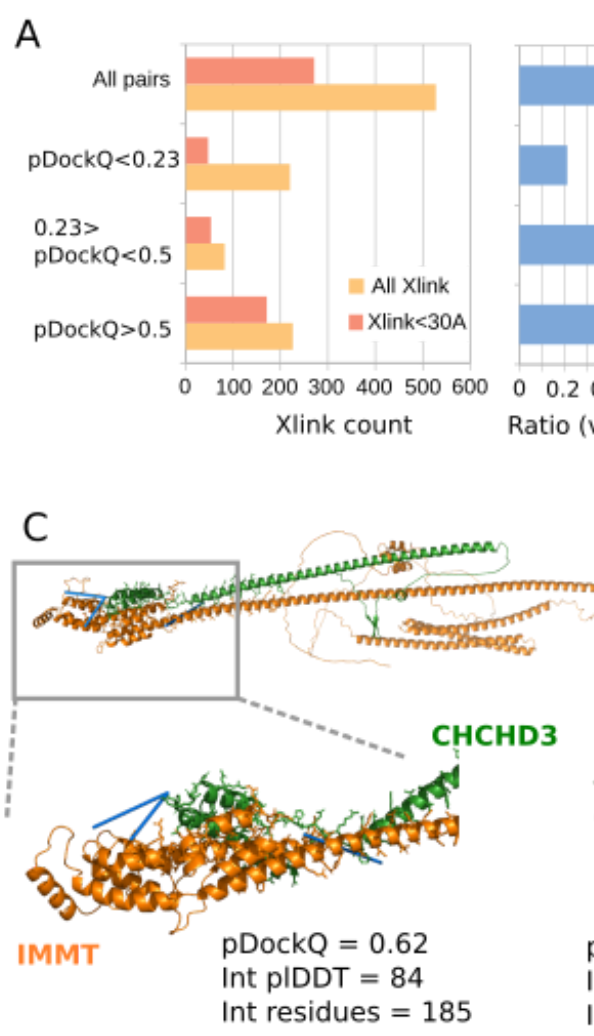
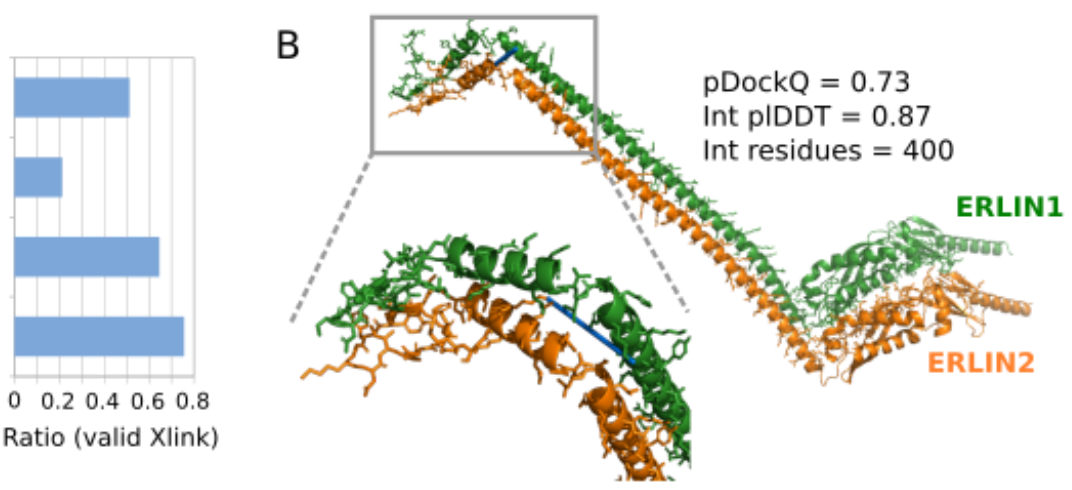

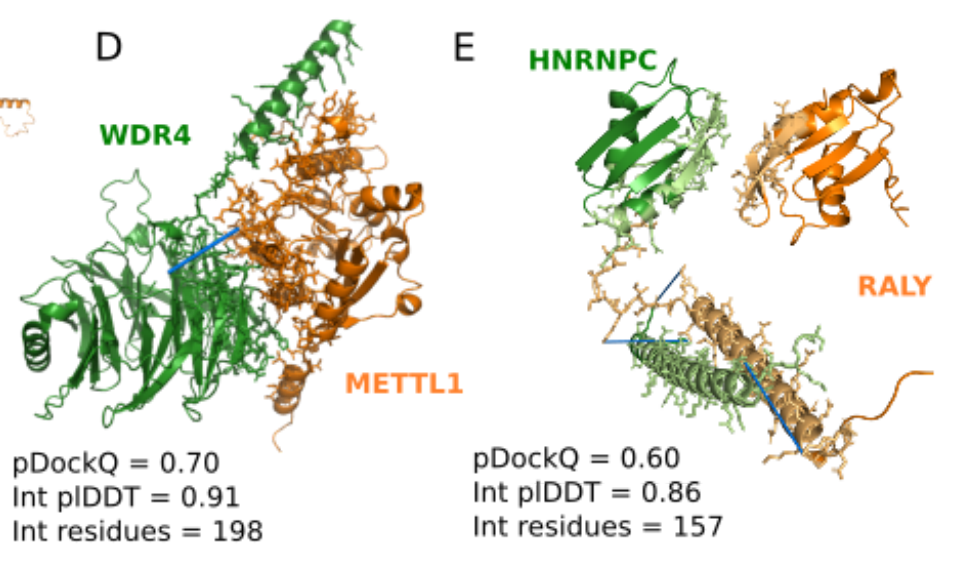

Figure 2 - Cross-link support for predicted complex structural models. A) The numbers and ratio of predicted structures having cross-link information for pairs of residues that bridge the two proteins in the predicted structure, broken down by the cross-links that satisfy their expected maximal distance and by the predicted quality of the model (pDockQ). B-E) Examples of predicted structures of high confidence, no prior structural information, and at least supported by one cross-link (indicated with blue line). 


\section{Disease-associated missense mutations at interfaces}

Missense mutations associated with human diseases can alter protein function via diverse mechanisms, including disrupting protein stability, allosterically modulating enzyme activity, and altering protein-protein interactions. Structural models can lead to the identification of interface residues allowing for the rationalisation of possible mechanisms of such interface disease mutations. To determine the usefulness of the predicted structures for studying disease mutations, we compiled a set of mutations located at interface residues experimentally tested for the impact on the corresponding interaction (IMEx Consortium Curators et al, 2019). We then performed in silico predictions of changes in binding affinity upon mutations using FoldX (Delgado et al, 2019) and observed that mutations known to disrupt the interactions are predicted to have a strong destabilisation of binding compared to mutations known not to have an effect (Fig. 3A). Higher confidence (pIDDT) of the mutated residues led to more substantial discrimination between mutations known to disrupt or not complex formation (Fig. 3A).

Having established the value of the predicted structures for modelling interface mutations, we mapped human disease (from ClinVar) and cancer mutations (from TCGA) to the interface residues defined by the set of high confidence protein complex predictions ( $\mathrm{pDockQ}>0.5)$. The hu.MAP and HuRI confident predictions identified 280 interfaces carrying pathogenic mutations and 602 interfaces corresponding to the top 25\% recurrently mutated interfaces in cancer, defined as the highest number of mutations per interface position (Fig. 3B, Methods). We illustrate in Fig. 3C examples of protein network clusters with interface disease mutations across a range of biological functions. For example, interface mutations in chromatin remodelling, including members of SWI/SNF complex (SMARCD1, SMARCD2, SMARCD3) and several transcription factors related to the development (e.g. TCF3 TCF4, LMO1 and LMO2). All of the disease mutation information is provided in Table S1.

We selected examples of interfaces with disease mutations and no previous experimental data or homology to available models (Fig. 3D-G). Fig. 3D shows the interface of WDR4-METTL1 that has supporting cross-link information described above. WDR4 has two annotated pathogenic variants at this interface, linked with Galloway-Mowat Syndrome 6, with the highlighted R170 participating in interactions with a negatively charged residue of METTL1. Fig. 3E shows an example of an interface with 32 recorded interface mutations in cancer for both proteins, including the highlighted argines in LDOC1, which form electrostatic interactions with the opposite chain. TWIST1 has several annotated pathogenic mutations, including L149R and $\mathrm{L} 159 \mathrm{H}$, which are at residues buried in the interface (Fig. 3F). In particular, the leucine to arginine mutation, associated with the Saethre-Chotzen syndrome, would strongly disrupt packing. The R118G mutation would disrupt the interaction with residue F22 mainchain $\mathrm{O}$ in TCF4. In RAD51D we found the mutation R266C (Breast-ovarian cancer, familial) that interacts across the interface with XRCC2 (Fig. 3F), paralogous genes involved in the repair of DNA double-strand breaks by homologous recombination. Interestingly, we also found mutations at R239, to Trp/GIn/Gly, associated with Breast-ovarian cancer that interacts with Tyr119 in XRCC2 that itself is also annotated as having mutations linked to hereditary cancer-predisposing syndrome. 

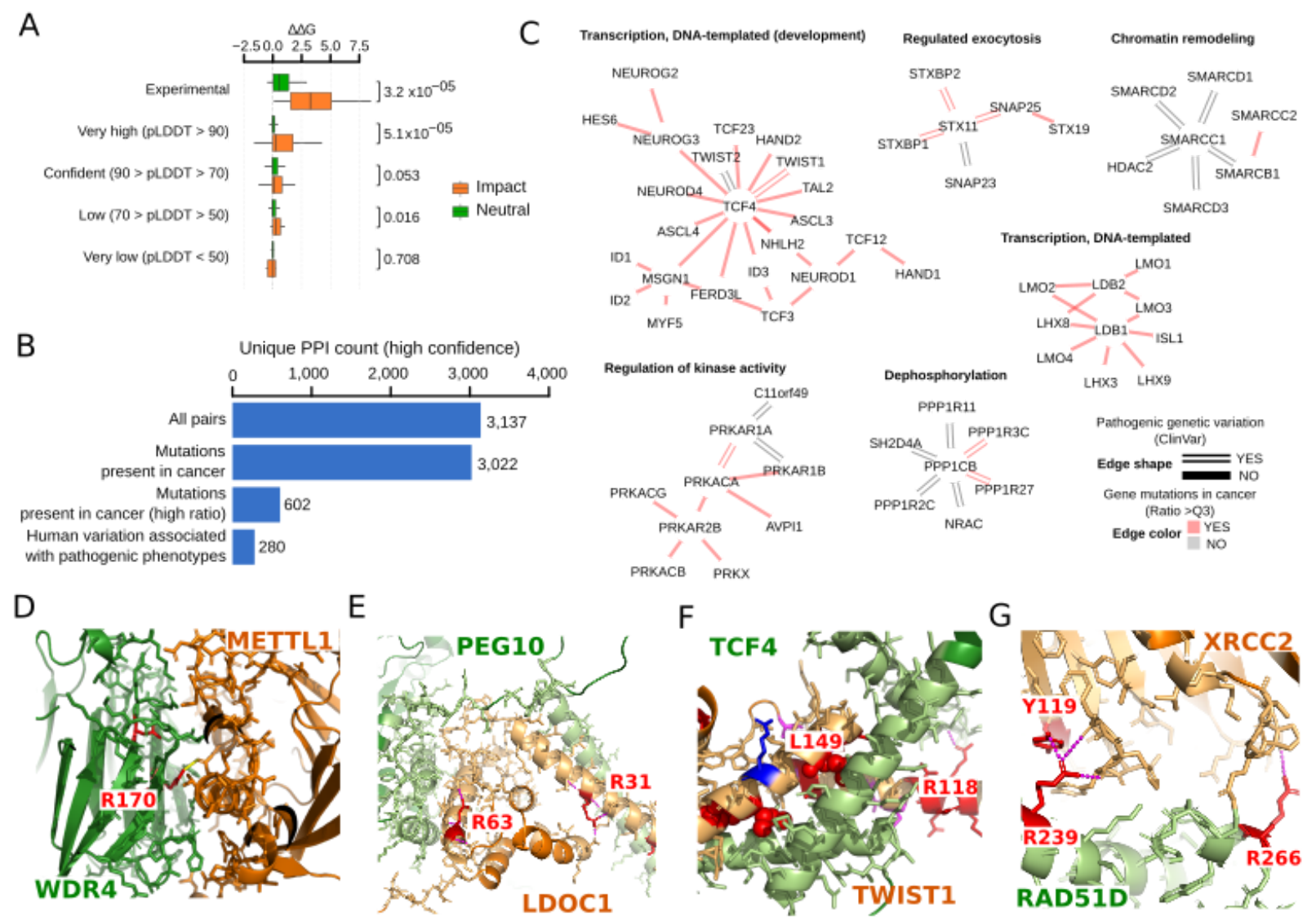

Figure 3 - Disease mutations at protein complex interface residues. A) Boxplot showing the distribution of $\Delta \Delta G$ for mutations known to have an impact (orange) versus the ones with neutral effect (green) B) unique protein-protein interaction pairs for high confidence models $(\mathrm{pDOCKQ}>0.5)$ in total, with mutations in cancer, mapped to the interface (all and top $25 \%$ ratios) and with pathogenic or likely pathogenic clinical variants mapped to the interface. C) Modules related to relevant biological processes. The colour of the edge represents the presence of cancer mutations in the interface (top $25 \%$ ratio, colour red) and the shape of the presence of pathogenic clinical variants (double line). D-G) Selected relevant structures with no prior structural knowledge showing clinical variants or mutations in cancer mapped to the interface (mutated residues in red).

\section{Phospho-regulation of protein complex interfaces}

Protein phosphorylation can regulate protein interactions by modulating the binding affinity via the change in size and charge of the modified residue. Over 100,000 experimentally human phosphorylation sites have been determined to date (Ochoa et al, 2020; Lawrence et al, 2016), but only 5 to $10 \%$ of these have a known function (Hornbeck et al, 2015). Mapping phosphorylation site positions to models of protein interfaces can generate mechanistic hypotheses for the functional role of phosphorylation sites in controlling protein interactions. We 
used a recent characterisation of the human phosphoproteome to identify 4145 unique phosphosites at interface residues of the set of confidently predicted structures. We noted that the average functional importance, defined by the functional score described by Ochoa and colleagues (Ochoa et al, 2020), was generally higher than random for phosphorylation sites at interfaces (Fig. 4A). Among the interface phosphorylation sites, we found some enrichment for targets of multiple kinases, including several MAPK pathway kinases part of the p38, JNK and ERK signalling pathways (Fig. 4B). This observation suggests that some interfaces in different protein pairs may be under coordinated regulation by specific kinases and conditions.

To identify potentially co-regulated interfaces, we collected measurements of changes in phosphorylation levels across a large panel of over 200 conditions (Ochoa et al, 2016). We retained 260 phosphosites that had a significant regulation in three conditions and then computed all-by-all pairwise correlations in phosphosite fold changes across conditions. We clustered these phosphosites by their profile of correlations (Fig. 4C), identifying 16 groups of co-regulated interface phosphorylation sites (Fig. 4C). For each group of phosphosites, we identified the conditions where these have the strongest up- or down-regulation and plotted a subset of conditions in Fig 4D. We also performed a GO enrichment analysis for each group of co-regulated phosphosites, including both proteins of the modified interfaces, to search for common biological functions (Fig 4E). For example, we observed a cluster of interface phosphosites in proteins related to intermediate filaments (cluster 7) that show strong regulation patterns along the cell cycle, downregulated in S-phase and up-regulated in G1 and mitosis. Phosphosites in cluster 1 (cell cycle G1-S phase transition) show the opposite trends with up-regulation in late S-phase and down-regulation in G1 and mitosis. Some clusters show regulation under specific kinase inhibition which may provide novel hypotheses for kinase regulation of specific processes. For example, phosphosites in cluster 9 (regulation of chromosome assembly) tend to be up-regulated after inhibition of ROCK and up-regulation after inhibition of mTOR.

While not all phosphosites at interfaces are likely to regulate the binding affinity, this analysis provides hypotheses for the potentially coordinated regulation of multiple proteins by tuning their interactions after specific perturbations. We provide the complete list of interface phosphosites, known kinase regulators and condition-specific regulation in Table S1. 
bioRxiv preprint doi: https://doi.org/10.1101/2021.11.08.467664; this version posted November 9, 2021. The copyright holder for this preprint (which was not certified by peer review) is the author/funder, who has granted bioRxiv a license to display the preprint in perpetuity. It is made available under aCC-BY-ND 4.0 International license.

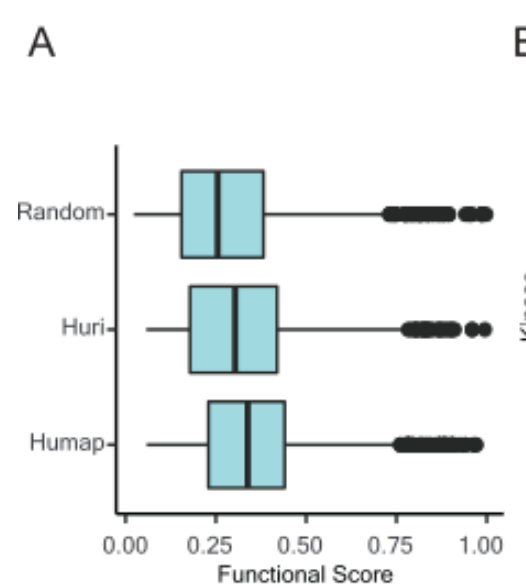

C

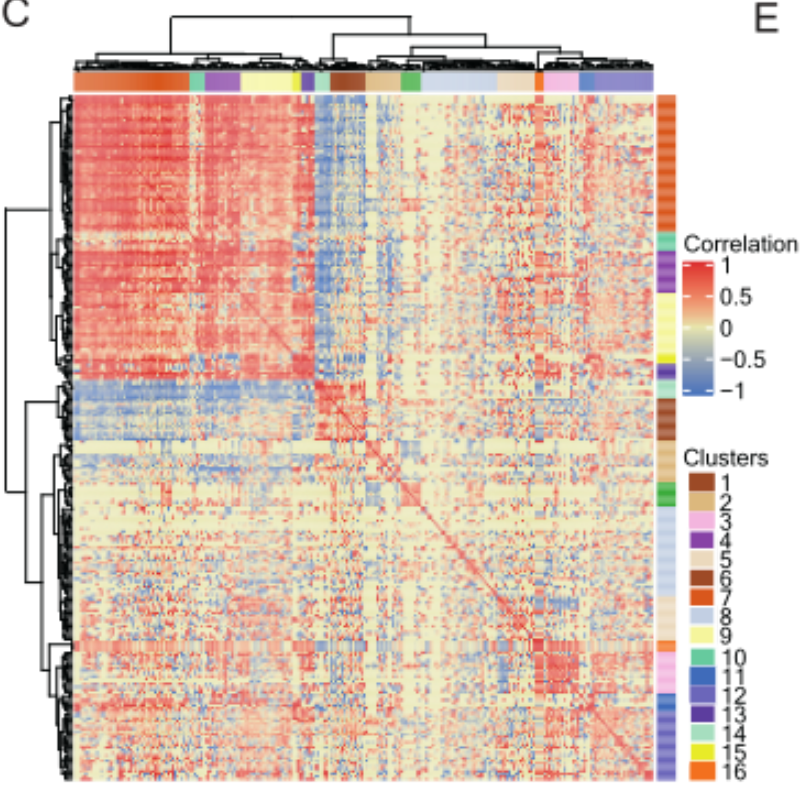

B

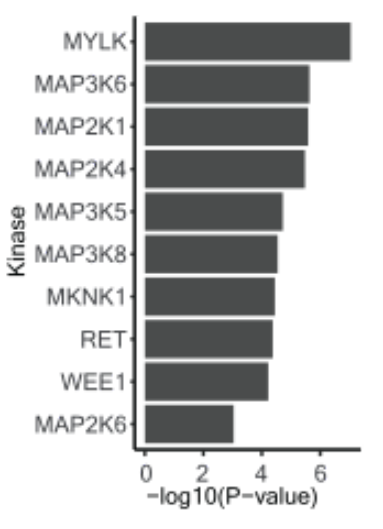

E
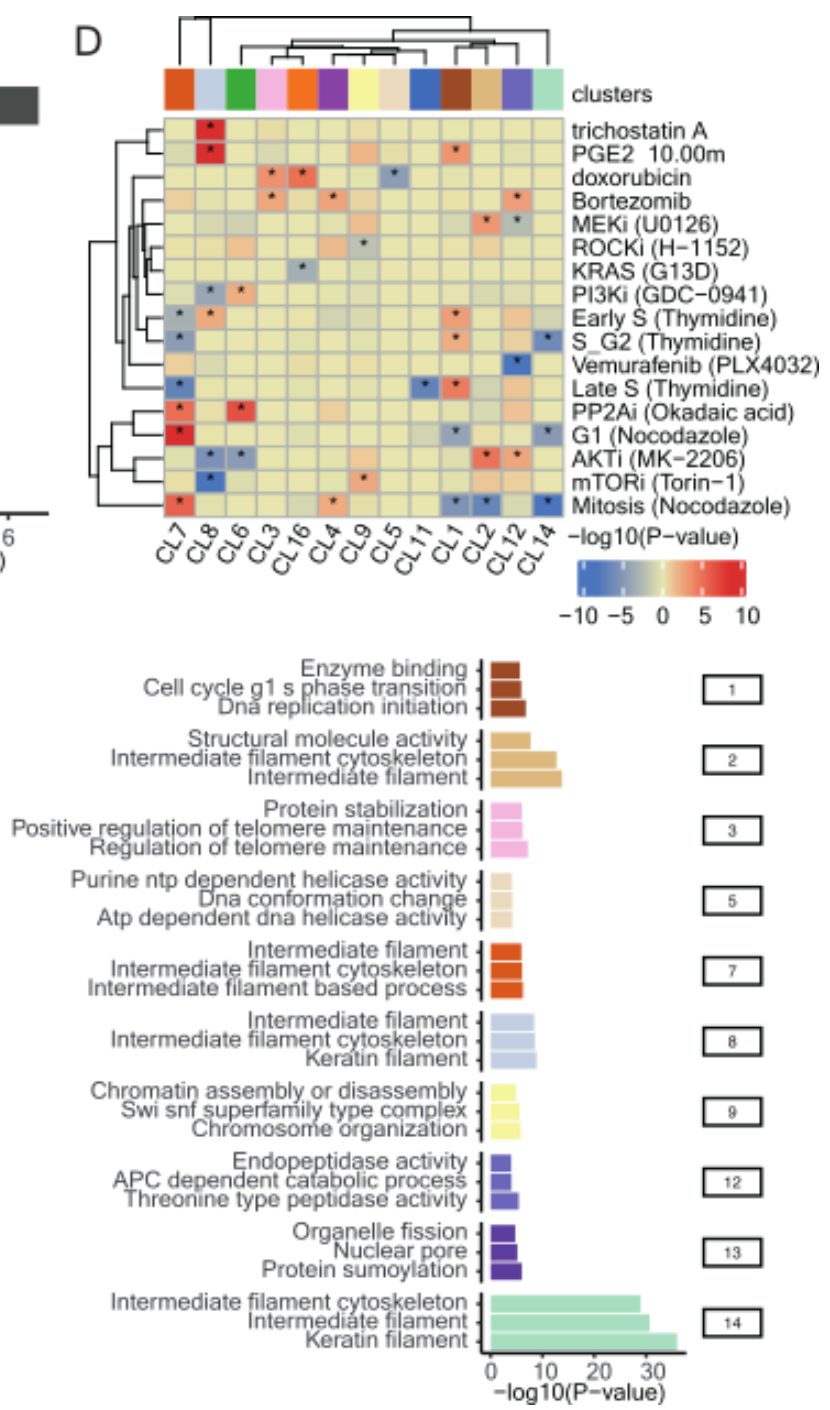

[ōob]

Figure 4 - Co-regulation of phosphorylation sites at interface residues. A) Distribution of phosphosite functional scores for phosphosites at interface residues and random phosphosites. B) Enrichment of kinase substrates among phosphosites at interface residues. C) Hierarchical clustering of the pairwise correlation values for changes in phosphosites levels across conditions. Groups of phosphosites showing high correlation values were defined as clusters (1 to 16) as indicated in colours along the outside of the clustergram. D) Degree of regulation of phosphosites from each cluster in a select panel of conditions, defined as a Z-test comparing the fold change of the phosphosites in a cluster compared with the entire distribution of fold changes in that condition. The result is summarized as the -log(P-value) and signed as positive if the median value is above the background or negative otherwise. E) Gene-ontology enrichment analysis for the proteins with phosphosites annotated to select clusters. 


\section{Higher-order assemblies of protein complexes from binary interactions}

Proteins interact with multiple partners either simultaneously, as part of larger protein complexes, or separated in time and space. This is also reflected in our structurally characterised network, where proteins can be found in groups as illustrated in a global network view of the interactions with confident models (Fig 5, central network). One key benefit of structurally characterising an interaction network is the identification of shared interfaces for multiple interactors. As an example, we highlight GDI1 (Rab GDP dissociation inhibitor alpha) that interacts with multiple Rab proteins regulating their activity by inhibiting the dissociation of GDP. The predicted complex structures for these interactions shows how these share the same interface and therefore cannot co-occur. Other clusters in the network suggest that the proteins form larger protein complex assemblies with many-to-many interactions. As the use of AlphaFold2 for predicting larger complex assemblies can be limited by computational requirements, we tested whether the structures for pairs of proteins could be iteratively structurally aligned. We tested this procedure on a small set of complexes covered in this network, with known structures and the number of subunits ranging from 5 (RFC complex, TFIIH core complex) to 14 (20S proteasome). We then aligned an experimentally determined structure with the predicted models (Fig. 5, grey - experimental model). These examples showcase the potential and also limitations of this procedure.

The TFIIH core complex is composed of 5 subunits with 1-to-1 stoichiometry. All subunits can be modelled with the final complex generally agreeing (Fig. 5) with a cryoEM structure for these subunits (6NMI). The most significant difference to the cryoEM model is the relative positioning of the ERCC3 subunit. The exact final model obtained can vary depending on the aligned pairs with multiple possible final conformations. Fig. 5 illustrates the conformation that best matches the cryoEM data. For example, for the TFIIH core complex, there is a predicted model where the complex adopts a more open conformation and alternative predicted placements of the GTF2H1 subunit.

The RFC complex is also composed of 5 subunits with 1-to-1 stoichiometry. One iterative alignment of pairwise interactions builds a model that includes all five subunits organised similarly to the observed in the 6VVO cryoEM structure (Fig. 5). In this predicted model, the subunits RFC2/5/4/3 match the experimentally observed model well, but there are apparent deviations introduced by compounding errors in alignment by this iterative process. Individual subunits in the cryoEM can be structurally aligned to each of the model subunits well, but then the alignment of the rest of the model is progressively worse the further away from the aligned subunit. The RFC1 subunit is individually not well predicted, showing a considerable difference between the cryoEM and Alphafold2 models. Some of the modelled pairs highlighted additional issues. For example, the RFC3 - RFC5 interaction pair is predicted with high confidence, while in fact, these do not share a direct contact in the experimental structure. AlphaFold2 places RFC3 at the RFC5-RFC4 interface, likely due to the structural similarity between RFC3 and RFC4. 
Encouraged by the examples tested, we defined an automatic procedure to generate larger models by iterative alignment of pairs (Methods). We start building all possible dimers in a complex, then sort them by $\mathrm{pDockQ}$, and start building from the first ranked dimers. Next, we add the highest-ranked dimer, which shares one subunit with the complex if it does not overlap; this is repeated for all dimers until the complex is complete or not more proteins can be added. We tested this on the $20 \mathrm{~S}$ proteasome, a particularly challenging example with stoichiometries different from 1-to-1 and homologous subunits. This automatic procedure could build a model containing all 14 subunits (half of the proteasome) that are mostly placed in agreement within the experimental model (Fig. 5). However, the exact order of the chains is incorrect, i.e. at each location an incorrect protein is placed, highlighting that AF2 can not distinguish which two proteins interact from a set of homologous proteins.

In summary, we find that it is possible to iteratively align structures of pairs of interacting proteins to build larger assemblies but identified issues that limit this procedure.

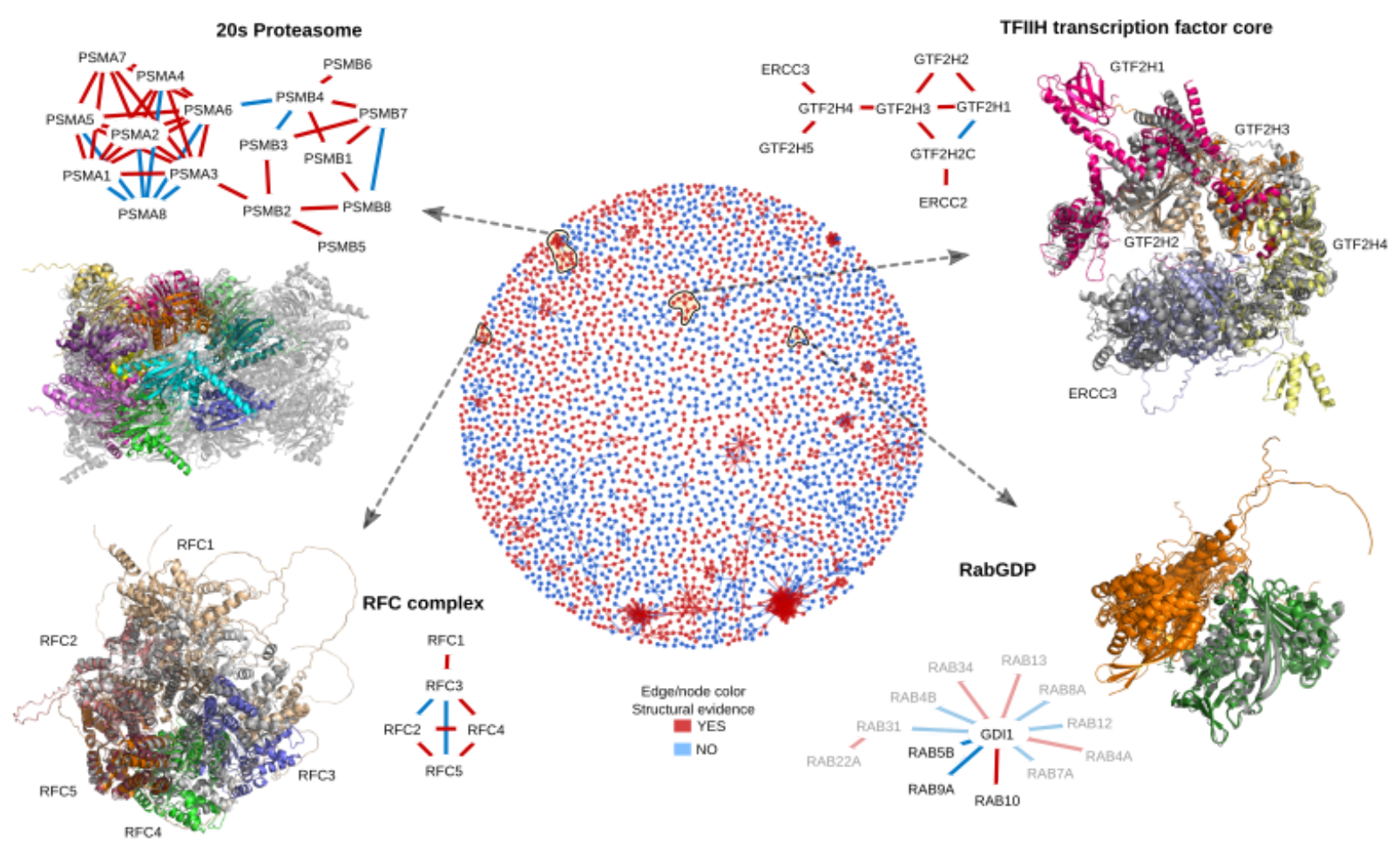

Figure 5 - Protein complexes for higher-order assemblies. The middle circle is a network view of all protein-protein interactions predicted with high confidence ( $p D o c k Q>0.5)$. The edges and nodes are coloured in red if there is a previous experimental or homology model for the interaction, in blue if such information is not available. We selected four examples of recapitulated complexes (yellow circles and black arrows) plotted in further detail. In these small networks, only the edges are coloured based on structural evidence. In the case of RabGDP the faded nodes and edges represent predictions with slightly lower confidence ( $p D o c k Q>0.3)$ 


\section{Discussion}

We have attempted here to generate predicted complex structures for pairs of human proteins known to physically interact from two different datasets based on different experimental approaches. We noted that the source of data used for the protein interactions is important and impacts the fraction of models that can be confidently predicted. Our analysis suggests the protein interactions supported by a combination of affinity-based and complementation based methods results in higher confidence models. We believe these interactions tend to correspond to high-affinity interactions that are very likely to share a direct physical permanent interaction. We show that it is possible to use metrics from the models (e.g. pDockQ score) to rank higher confidence models, providing an additional accuracy level to the large scale protein-protein interaction studies. Further, future large-scale computational predictions of protein-protein interactions may provide additional high-quality targets for detailed studies of stable complexes. Experimental data from cross-link mass spectrometry experiments provide an ideal resource for further validating these predictions via orthogonal means. In principle, such constraints from cross-link could also be considered during predictions, and it may be possible in the future to develop predictors that can take in such constraints as part of the starting information.

Based on comparisons with solved structures, we suggest that models with $\mathrm{pDockQ}>0.5$ are very likely to be correct. Additionally, models with lower scores $(0.5>p D o c k Q>0.23)$ are still likely to contain many correct solutions and may highlight correct interfaces even if not correct orientations of the interacting proteins. In this study this would correspond to an additional 6000 complex structures. Such lower confidence models are likely to be useful for generating hypotheses and large-scale analysis of global properties. Equally important is the caveat that high confidence predictions will still contain errors, and in particular, we note that in protein complexes containing homologous proteins, the current procedure cannot identify the exact pairing of the protein. For such cases, additional methods need to be developed.

Structural models for protein interfaces are critical for understanding molecular mechanisms and the impact of mutations and post-translational modifications. We illustrate this using disease mutations and phosphorylation data. While much disease-associated variation is often found in non-coding regions of the genome, the growth of exome sequencing of large cohorts of patients will lead to discovering many more protein mutations linked to disease, which will require such large structural characteristics. Both for mutations and phosphorylation sites, we think these analyses should be seen as generating hypotheses for further testing, and we make this information available in the supplementary material to facilitate such future work.

Finally, in principle, we show that it is possible to build structural models for larger assemblies from the binary complexes predicted here. Aspects that may limit this include the structural homology between subunits, unknown subunit stoichiometries and multiple possible predicted conformations. Additional work will be needed to design methods and to score systems to build such larger complex assemblies. 


\section{Methods}

\section{Protein interaction data and annotations}

Human protein pairs known to physically interact were obtained from the Hu.MAP dataset, retaining pairwise interactions with $>=0.5$ confidence, and most interactions from the HuRI dataset. These interactions were further enriched by obtaining annotations on cross-linking peptides matched across pairs of interactions, disease related mutations and protein phosphorylation sites in the selected proteins. Crosslink data was collected from (Yugandhar et al, 2020; Schweppe et al, 2016; Klykov et al, 2020; Steigenberger et al, 2019; Klykov et al, 2018; Fasci et al, 2018; Eliseev et al, 2018; Gestaut et al, 2019; Klatt et al, 2020; Sabath et al, 2020; Mohamed et al, 2021), filtered for peptides assigned to only one sequence. Clinical missense variants associated with disease were collected from ClinVar. We selected only those having pathogenic or likely pathogenic effects which were mapped to Uniprot protein sequences using VarMap. The final list of mutated positions was then compared to the interface positions. We obtained a list of protein phosphorylation sites with predicted functional relevance (Ochoa et al, 2020), phosphosite annotations (Hornbeck et al, 2015) and regulation of phosphorylation sites across a large panel of conditions (Ochoa et al, 2016). These phosphosites were also mapped to interface positions as defined by the predicted models. All protein interaction networks were processed using $R$ packages igraph (v1.2.5) and qgraph ( $v$ 1.9), further aesthetics improvements were done using Cytoscape (Shannon et al, 2003).

\section{Protein complex prediction}

To predict protein complexes of pairwise interactions, we utilise the FoldDock pipeline (Bryant et al, 2021) based on AlphaFold2 (Jumper et al, 2021). We use the option of fused+paired multiple sequence alignments (MSAs) and run the model configuration m1-10-1 as this provides the highest success rate accompanied by a 20 -fold speed-up. Both the fused and paired MSAs are constructed from running $\mathrm{HHblits}$ on every single chain against Uniclust 30 . The fused MSA is generated by simply concatenating the output of each of the single-chain HHblits runs for two interacting chains. The paired MSA is constructed by combining the top hit for each matching OX identifier between two interacting chains, using the output from the single-chain HHblits runs.

\section{pDockQ confidence score}

To score models, we use features from the predicted complexes to calculate the predicted DockQ score, $p D o c k Q$. This score is defined with the following sigmoidal equation: 
$p D o c k Q=\frac{0.707}{1+e^{-0.03148(x-388.06)}}+0.03138$

where,

$x=$ average interface $\mathrm{pIDDT} \cdot \log ($ number of interface contacts).

The parameters were optimised to predict the DockQ score using the dataset from (Green et al, 2021). The number of interface contacts is defined as elsewhere in this paper (any residues with an interface atom within $10 \AA$ to the other chain), and the pIDDT is the predicted IDDT score from AlphaFold2 taken over the interface residues as defined by the interface contacts.

\section{Building larger complexes from binary interactions}

A simple procedure to build larger complexes from a set of paired models was developed. All dimers in the set are by default ranked by their $\mathrm{pDockQ}$ values.

1. The building is started from a single dimer by default the dimer with the highest pDockQ value. This is referred to as the "complex".

2. All other dimers in the set are then tried to be added to the "complex. Starting with the one with the second highest $\mathrm{PDock} Q$ a chain is added to the complex if:

a. Exactly one chain of the dimer is identical to one chain in the complex

b. The structure of these two chains is similar enough (default TM-score $>0.8$ )

c. The dimer is then rotated so that the two chains overlap-

d. The second chain in the dimer does not clash with more than $25 \%$ of its residues $(C A<5 \AA)$ to any chain in the complex.

3. If a chain is added, the procedure is started over again and repeated until no more chains can be added.

\section{Analysis of phosphosites in the protein-protein interfaces}

Phosphosite residues in interfaces were identified from a previously published comprehensive list of known human phosphosites (Ochoa et al, 2020). Kinases associated with phosphorylation of interface residues were obtained from the PhosphositePlus database and over-representation analysis of kinases was performed using a hyper-geometric test. Highly regulated interface phosphosites were defined as those with more than two-fold change in phosphorylation in more than two perturbation conditions across a collated phosphoproteomics dataset comprising a range of physiological conditions and drug treatments (Ochoa et al, 2016). Pearson correlation was calculated amongst these regulated phosphosites and clusters of co-regulated phosphosites were identified using hierarchical clustering ('ward' method) of euclidean distances of the correlation matrix. Phosphosite clusters were created by cutting the dendrogram at the appropriate level using cutree $(\mathrm{h}=17)$ function in $\mathrm{R}$. Phosphosite clusters that were significantly regulated in each perturbation condition were identified by z-test from the 
comparison of fold changes in phosphosite measurements of all phosphosites in a cluster against the overall distribution of phosphorylation fold changes across the condition. Gene ontology over-representation of each cluster was performed separately using a hypergeometric test in R. The gene ontology terms were obtained from the c5 category of Molecular Signature Database (MSigDBv7.1) (Subramaniam et al, 2005). All over-representation analysis (ORA) were performed using the enricher function of clusterProfiler package (version 3.12.0) (Wang et al, 2012) in R.

\section{Availability}

All code used in this project can be found at https://gitlab.com/ElofssonLab/huintaf2/. Tools to run AlphaFold2 can be found at https://gitlab.com/ElofssonLab/FoldDock/. All models generated as well as some of the multiple sequence alignments can be found at https://archive.bioinfo.se/huintaf2/.

\section{Acknowledgements}

R.A.S. acknowledges funding through the European Union Horizon 2020 program INFRAIA project Epic-XS (Project 823839) and the research programme NWO TA with project number 741.018.201, which is partly financed by the Dutch Research Council (NWO). AE was funded by the Vetenskapsrådet Grant No. 2016-03798 and Knut and Alice Wallenberg foundation. Computational resources were provided by SNIC, grant No: SNIC 2021/5-297 and Berzelius-2021-29.

\section{References}

Akdel M, Pires DEV, Pardo EP, Jänes J, Zalevsky AO, Mészáros B, Bryant P, Good LL, Laskowski RA, Pozzati G, et al (2021) A structural biology community assessment of AlphaFold 2 applications. bioRxiv: 2021.09.26.461876

Baek M, DiMaio F, Anishchenko I, Dauparas J, Ovchinnikov S, Lee GR, Wang J, Cong Q, Kinch LN, Dustin Schaeffer R, et al (2021) Accurate prediction of protein structures and interactions using a three-track neural network. Science 373: 871-876 doi:10.1126/science.abj8754 [PREPRINT]

Beltrao P, Albanèse V, Kenner LR, Swaney DL, Burlingame A, Villén J, Lim WA, Fraser JS, Frydman J \& Krogan NJ (2012) Systematic functional prioritization of protein posttranslational modifications. Cell 150: 413-425

Betts MJ, Wichmann O, Utz M, Andre T, Petsalaki E, Minguez P, Parca L, Roth FP, Gavin A-C, Bork P, et al (2017) Systematic identification of phosphorylation-mediated protein interaction switches. PLoS Comput Biol 13: e1005462 
Bryant P, Pozzati G \& Elofsson A (2021) Improved prediction of protein-protein interactions using AlphaFold2. bioRxiv: 2021.09.15.460468

Burley SK, Bhikadiya C, Bi C, Bittrich S, Chen L, Crichlow GV, Christie CH, Dalenberg K, Di Costanzo L, Duarte JM, et al (2021) RCSB Protein Data Bank: powerful new tools for exploring 3D structures of biological macromolecules for basic and applied research and education in fundamental biology, biomedicine, biotechnology, bioengineering and energy sciences. Nucleic Acids Res 49: D437-D451

Cong Q, Anishchenko I, Ovchinnikov S \& Baker D (2019) Protein interaction networks revealed by proteome coevolution. Science 365: 185-189

Delgado J, Radusky LG, Cianferoni D \& Serrano L (2019) FoldX 5.0: working with RNA, small molecules and a new graphical interface. Bioinformatics 35: 4168-4169

Drew K, Wallingford JB \& Marcotte EM (2021) hu.MAP 2.0: integration of over 15,000 proteomic experiments builds a global compendium of human multiprotein assemblies. Mol Syst Biol 17: e10016

Eliseev B, Yeramala L, Leitner A, Karuppasamy M, Raimondeau E, Huard K, Alkalaeva E, Aebersold R \& Schaffitzel C (2018) Structure of a human cap-dependent 48S translation pre-initiation complex. Nucleic Acids Res 46: 2678-2689

Evans R, O’Neill M, Pritzel A, Antropova N, Senior A, Green T, Žídek A, Bates R, Blackwell S, Yim J, et al (2021) Protein complex prediction with AlphaFold-Multimer. bioRxiv: 2021.10.04.463034

Fasci D, van Ingen H, Scheltema RA \& Heck AJR (2018) Histone Interaction Landscapes Visualized by Crosslinking Mass Spectrometry in Intact Cell Nuclei. Mol Cell Proteomics 17: 2018-2033

Gestaut D, Roh SH, Ma B, Pintilie G, Joachimiak LA, Leitner A, Walzthoeni T, Aebersold R, Chiu W \& Frydman J (2019) The Chaperonin TRiC/CCT Associates with Prefoldin through a Conserved Electrostatic Interface Essential for Cellular Proteostasis. Cell 177: 751-765.e15

Green AG, Elhabashy H, Brock KP, Maddamsetti R, Kohlbacher O \& Marks DS (2021) Large-scale discovery of protein interactions at residue resolution using co-evolution calculated from genomic sequences. Nat Commun 12: 1396

Hornbeck PV, Zhang B, Murray B, Kornhauser JM, Latham V \& Skrzypek E (2015) PhosphoSitePlus, 2014: mutations, PTMs and recalibrations. Nucleic Acids Res 43: D512-20

Humphreys IR, Pei J, Baek M, Krishnakumar A, Anishchenko I, Ovchinnikov S, Zhang J, Ness TJ, Banjade S, Bagde S, et al (2021) Structures of core eukaryotic protein complexes. bioRxiv: 2021.09.30.462231

IMEx Consortium Curators, Del-Toro N, Duesbury M, Koch M, Perfetto L, Shrivastava A, Ochoa D, Wagih O, Piñero J, Kotlyar M, et al (2019) Capturing variation impact on molecular interactions in the IMEx Consortium mutations data set. Nat Commun 10: 10

Jumper J, Evans R, Pritzel A, Green T, Figurnov M, Ronneberger O, Tunyasuvunakool K, Bates 
R, Žídek A, Potapenko A, et al (2021) Highly accurate protein structure prediction with AlphaFold. Nature 596: 583-589

Kamburov A, Lawrence MS, Polak P, Leshchiner I, Lage K, Golub TR, Lander ES \& Getz G (2015) Comprehensive assessment of cancer missense mutation clustering in protein structures. Proc Natl Acad Sci U S A 112: E5486-95

Klatt F, Leitner A, Kim IV, Ho-Xuan H, Schneider EV, Langhammer F, Weinmann R, Müller MR, Huber R, Meister $\mathrm{G}$, et al (2020) A precisely positioned MED12 activation helix stimulates CDK8 kinase activity. Proc Natl Acad Sci U S A 117: 2894-2905

Klykov O, Steigenberger B, Pektaş S, Fasci D, Heck AJR \& Scheltema RA (2018) Efficient and robust proteome-wide approaches for cross-linking mass spectrometry. Nat Protoc 13: 2964-2990

Klykov O, van der Zwaan C, Heck AJR, Meijer AB \& Scheltema RA (2020) Missing regions within the molecular architecture of human fibrin clots structurally resolved by XL-MS and integrative structural modeling. Proc Natl Acad Sci U S A 117: 1976-1987

Lawrence RT, Searle BC, Llovet A \& Villén J (2016) Plug-and-play analysis of the human phosphoproteome by targeted high-resolution mass spectrometry. Nat Methods 13: 431-434

Luck K, Kim D-K, Lambourne L, Spirohn K, Begg BE, Bian W, Brignall R, Cafarelli T, Campos-Laborie FJ, Charloteaux B, et al (2020) A reference map of the human binary protein interactome. Nature 580: 402-408

Mohamed WI, Park SL, Rabl J, Leitner A, Boehringer D \& Peter M (2021) The human GID complex engages two independent modules for substrate recruitment. EMBO Rep 22: e52981

Mosca R, Céol A \& Aloy P (2012) Interactome3D: adding structural details to protein networks. Nat Methods 10: 47-53

Mosca R, Céol A, Stein A, Olivella R \& Aloy P (2014) 3did: a catalog of domain-based interactions of known three-dimensional structure. Nucleic Acids Res 42: D374-9

Nishi H, Hashimoto K \& Panchenko AR (2011) Phosphorylation in protein-protein binding: effect on stability and function. Structure 19: 1807-1815

Ochoa D, Jarnuczak AF, Viéitez C, Gehre M, Soucheray M, Mateus A, Kleefeldt AA, Hill A, Garcia-Alonso L, Stein F, et al (2020) The functional landscape of the human phosphoproteome. Nat Biotechnol 38: 365-373

Ochoa D, Jonikas M, Lawrence RT, El Debs B, Selkrig J, Typas A, Villén J, Santos SD \& Beltrao $P$ (2016) An atlas of human kinase regulation. Mol Syst Biol 12: 888

Orchard S, Ammari M, Aranda B, Breuza L, Briganti L, Broackes-Carter F, Campbell NH, Chavali G, Chen C, del-Toro N, et al (2014) The MlntAct project--IntAct as a common curation platform for 11 molecular interaction databases. Nucleic Acids Res 42: D358-63

Porta-Pardo E, Garcia-Alonso L, Hrabe T, Dopazo J \& Godzik A (2015) A Pan-Cancer Catalogue of Cancer Driver Protein Interaction Interfaces. PLoS Comput Biol 11: e1004518 
Pozzati G, Zhu W, Bassot C, Lamb J, Kundrotas P \& Elofsson A Limits and potential of combined folding and docking using PconsDock. doi:10.1101/2021.06.04.446442 [PREPRINT]

Sabath K, Stäubli ML, Marti S, Leitner A, Moes M \& Jonas S (2020) INTS10-INTS13-INTS14 form a functional module of Integrator that binds nucleic acids and the cleavage module. Nat Commun 11: 3422

Schweppe DK, Zheng C, Chavez JD, Navare AT, Wu X, Eng JK \& Bruce JE (2016) XLinkDB 2.0: integrated, large-scale structural analysis of protein crosslinking data. Bioinformatics 32: $2716-2718$

Shannon P, Markiel A, Ozier O, Baliga NS, Wang JT, Ramage D, Amin N, Schwikowski B \& Ideker T (2003) Cytoscape: a software environment for integrated models of biomolecular interaction networks. Genome Res 13: 2498-2504

Šoštarić N, O’Reilly FJ, Giansanti P, Heck AJR, Gavin A-C \& van Noort V (2018) Effects of Acetylation and Phosphorylation on Subunit Interactions in Three Large Eukaryotic Complexes. Mol Cell Proteomics 17: 2387-2401

Steigenberger B, Pieters RJ, Heck AJR \& Scheltema RA (2019) PhoX: An IMAC-Enrichable Cross-Linking Reagent. ACS Cent Sci 5: 1514-1522

Subramaniam V, Vincent IR \& Jothy S (2005) Upregulation and dephosphorylation of cofilin: Modulation by CD44 variant isoform in human colon cancer cells. Experimental and Molecular Pathology 79: 187-193 doi:10.1016/j.yexmp.2005.08.004 [PREPRINT]

Wang X, Wei X, Thijssen B, Das J, Lipkin SM \& Yu H (2012) Three-dimensional reconstruction of protein networks provides insight into human genetic disease. Nat Biotechnol 30: 159-164

Yugandhar K, Wang T-Y, Leung AK-Y, Lanz MC, Motorykin I, Liang J, Shayhidin EE, Smolka MB, Zhang S \& Yu H (2020) MaXLinker: Proteome-wide Cross-link Identifications with High Specificity and Sensitivity. Mol Cell Proteomics 19: 554-568

Zhang QC, Petrey D, Deng L, Qiang L, Shi Y, Thu CA, Bisikirska B, Lefebvre C, Accili D, Hunter $\mathrm{T}$, et al (2012) Structure-based prediction of protein-protein interactions on a genome-wide scale. Nature 490: 556-560 